\title{
A temporary threshold shift for self-motion detection following sustained, oscillating linear acceleration
}

\author{
D. E. PARKER, W. L. GULlEDGE, R. L. TUBBS, and V. M. LITTLEFIELD \\ Miami University, Oxford, Ohio 45056
}

\begin{abstract}
Thresholds for detecting linear motion (self-motion) increased following exposure of human observers to sustained linear oscillation (fatiguing stimulus) at $0.26 \mathrm{~Hz}$ and approximately $0.5 \mathrm{G}$ peak-to-peak for durations up to $30 \mathrm{~min}$. Recovery to preexposure levels took place over a period of 10 to $15 \mathrm{~min}$ following the sustained oscillation termination. Differences in threshold shift magnitudes following sustained oscillation in various orientations support the interpretation that the observed threshold shifts resulted from fatigue of the utricular otolith receptors. Threshold shifts were not obtained following exposure of the human observers to sustained, unidirectional linear acceleration $(2 \mathrm{G})$ for durations up to $10 \mathrm{~min}$ with a centrifuge. The results of this study suggest a previously unobserved response property of the otolith organs, namely, that these organs can be fatigued in a manner analogous to other receptor systems.
\end{abstract}

The experiments described in this paper examined changes in thresholds for detection of displacement by human observers following exposure to sustained linear oscillation or to centrifugation. These experiments can be understood within the general framework of research concerning vestibular plasticity. Four categories of vestibular plasticity may be suggested: fatigue, adaptation, habituation, and sensorymotor learning. Adaptation has been investigated both behaviorally (e.g., Schöne \& Lechner-Steinleitner, 1978) and neurophysiologically (e.g., Fernandez \& Goldberg, 1976). Habituation studies for both animals and human beings have recently been summarized by Collins (1974a, 1974b). Sensory-motor learning has been investigated employing a slowly rotating room (e.g., Reason \& Graybiel, 1970). In contrast to the extensive investigation of adaptation, habituation, and sensory-motor learning, vestibular studies yielding data that might be interpreted as indicating fatigue have not been performed previously.

The experiments which follow were modeled on the fatigue studies that have been pursued in the field of auditory research. One set of studies from the auditory fatigue literature involves investigation of the temporary threshold shifts (TTS) induced by

This investigation was supported by funds from Contract F3361575C5029 between the United States Air Force and Miami University, Contract NAS 914538 between the National Aeronautics and Space Administration and Miami University, and the Miami University Faculty Research Committee. We thank F. E. Guedry, C. S. Harris, and H. Schöne for their comments on a preliminary draft of this paper. We thank also the observers who maintained a high level of attention and interest despite quite uncomfortable working conditions. Requests for reprints should be addressed to Donald E. Parker, Department of Psychology, Miami University, Oxford, Ohio 45056. intense sound exposure. Thresholds for detecting sound are determined before and after exposure of the observer to intense auditory stimulation. Generally, the amplitude of the auditory threshold increases with increasing durations of intense sound exposure and the recovery time is proportional to the size of the initial threshold shift. These results have been interpreted as indicating fatigue of the auditory receptor. The studies that follow were designed to test the hypothesis that a vestibular TTS, analogous to an auditory TTS and interpretable as indicating vestibular fatigue, could be observed following sustained vestibular stimulation.

\section{EXPERIMENT 1 THRESHOLD SHIFT}

\section{Method}

Experiment 1 was designed to determine whether linear motion detection thresholds could be altered by exposure of observers to sustained linear oscillation (fatiguing stimulus).

Observers. Six graduate students who reported no previous auditory or vestibular difficulties and who were naive concerning the purpose of the experiment and the characteristics of the vestibular system served as observers. They were chosen primarily because they were the appropriate height and weight to fit into the parallel swing restraint. Three were males and three were females; the age range was 22 to 26 years.

Apparatus. The stimulus apparatus was a parallel swing which has been described elsewhere (Parker, 1970). This parallel swing consisted of a flat bed suspended from the ceiling by four steel cables, $3.74 \mathrm{~m}$ in length. The bed of the swing was $2.4 \mathrm{~m}$ long and $1 \mathrm{~m}$ wide. This bed supported a metal cylinder that was $1.8 \mathrm{~m}$ long and had a diameter of $1.97 \mathrm{~m}$. A Styrofoam negative body mold designed to enclose a standard 73-kg man was located inside the cylinder. The entire cylinder, within which the observer was restrained, could be rotated to any position around the observer's longitudinal axis. A head restraint system and bite-board were attached to the cylinder. In the $0^{\circ}$ orientation, the main parts of 
the observer's utricular maculae were in the horizontal plane with the otoliths above the maculae. In the $90^{\circ}$ orientation, the main parts of the utricular maculae were in the vertical plane, and in the $180^{\circ}$ orientation, the main parts of the utricules were in the horizontal plane with the otoliths suspended below the maculae. Linear acceleration amplitude was determined with a Statham accelerometer and a Grass polygraph; the accelerometer was attached to the parallel swing bed.

Procedure. The observer was placed in the prone position on the body-contoured Styrofoam mold in the parallel swing cylinder, padded with foam rubber to insure a snug fit, and locked into the cylinder. The bite-board and head holder were adjusted so that the observer's head was held securely with the main parts of the utricular maculae in the horizontal plane $\left(0^{\circ}\right.$ orientation). A wooden box was placed over the head end of the parallel swing, eliminating light cues and air currents. The observer was instructed to keep his eyes closed during the experiment to further reduce the possibility that visual cues might be used to detect motion.

The experimenter, who served as the parallel swing "motor," pushed the swing slowly away from himself and then pulled the swing to the desired displacement position. The swing was held stationary at the desired displacement for 2-3 sec prior to smooth release. For threshold trials, the swing was allowed to complete one oscillation before the experimenter asked the observer to indicate whether he detected motion. This procedure was followed to reduce the likelihood that the observer responded to some transient motion associated with swing release. For extended periods of oscillation, the experimenter gently pushed the swing intermittently to maintain the desired displacement amplitude. Displacement amplitudes were determined by noting the relationship between a pointer attached to the swing and a scale taped to the floor.

Each experimental session consisted of three parts: (1) prefatigue threshold determination, (2) exposure to sustained oscillation (fatiguing stimulus) for one of four durations $(0,2,5$, or $10 \mathrm{~min})$, and (3) postfatigue threshold determination. For the 0 -min condition, there was a 5-min control interval, during which the parallel swing remained motionless, between the pre- and postfatigue threshold determinations.

Motion detection thresholds were determined employing a tracking procedure. The parallel swing was set in motion at a particular displacement amplitude, usually between 4 and $8 \mathrm{~cm}$ peak-topeak. The experimenter pressed a microswitch that produced a light flash in the wooden box whenever he wanted the observer's estimation of whether the parallel swing was in motion or not. This flash was easily detected by the observer even though his eyes were closed. If the observer felt motion, he responded by pressing his microswitch, which illuminated a lamp that could be seen by the experimenter. If the observer failed to detect motion, the swing was stopped and then set in motion at a displacement amplitude $1 \mathrm{~cm}$ greater; alternatively, if the observer did detect motion, the swing was stopped and then set in motion at a displacement amplitude $1 \mathrm{~cm}$ smaller. The displacement amplitude at which a reversal (change from increasing to decreasing displacement or vice versa) occurred was recorded as a threshold. Tracking continued until 10 reversals had been completed. The average displacement amplitude for the 10 reversals was recorded as the observer's threshold for the particular condition.

Catch trials, in which observers were asked to indicate whether they felt movement when there was in fact no movement, were employed at random intervals to insure that observers were not reporting "phantom" movement or aftereffects.

Fatigue was induced by oscillating the parallel swing at $.26 \mathrm{~Hz}$ with a peak-to-peak displacement of $176 \mathrm{~cm}$, which produced peak-to-peak accelerations of $468 \mathrm{~cm} / \mathrm{sec}^{2}$. Each observer was exposed to each of the four fatiguing stimulus durations four times for a total of 16 experimental sessions. The order of fatiguing stimulus durations was randomized across observers and sessions. At least $24 \mathrm{~h}$ elapsed between sessions.

Postfatigue thresholds were determined in the same manner as prefatigue thresholds, beginning approximately $1 \mathrm{~min}$ after fatigue termination. A period of about 4 min was required to complete the 10 postfatigue threshold determinations.

\section{Results}

Frgure 1 illustrates the results of Experiment 1. The ordinate indicates changes in the threshold for detection of motion (threshold shift) as a function of fatiguing stimulus duration (abscissa). The curve demonstrates a monotonic relationship between threshold shift and fatiguing stimulus duration. The fact that the threshold shift was greater following an increase in fatiguing stimulus duration from 2 to 5 min than following the increase in duration from 5 to $10 \mathrm{~min}$ indicates a negatively accelerated relationship between threshold shift and fatiguing stimulus duration. Differences among absolute threshold levels as a function of fatiguing stimulus duration $(0,2,5$, and $10 \mathrm{~min})$ were statistically significant, $\mathrm{F}(3,15)=31.0, \mathrm{p}<.0001$.

\section{Discussion}

The results of Experiment 1 indicated that motion detection thresholds could be increased by exposing observers to sustained linear oscillation. The fact that the observers' prefatigue thresholds were nearly constant across days indicated that the threshold shifts obtained in Experiment 1 were temporary. Experiment 2 was undertaken to determine the time course of the recovery from this temporary threshold shift.

\section{EXPERIMENT 2 RECOVERY}

\section{Method}

Experiment 2 was essentially a repetition of Experiment 1 with the following exceptions. Three (two females and one male) rather

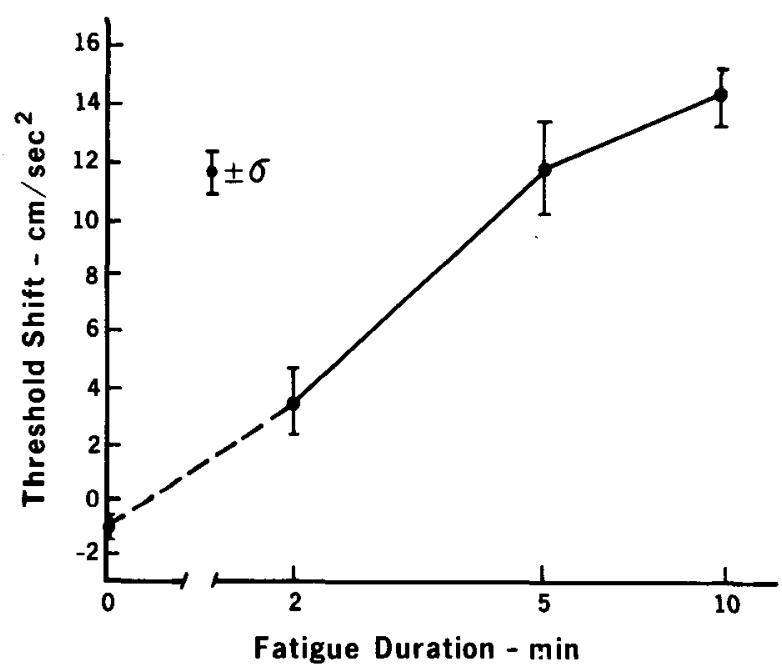

Figure 1. Shifts in linear motion detection thresholds following sustained oscillation (fatiguing stimulus) of varying durations. Threshold shifts are specified in parallel swing peak-to-peak acceleration- $\mathrm{cm} / \mathrm{sec}^{2}$. The fatiguing stimulus was $468 \mathrm{~cm} / \mathrm{sec}^{2}$ peakto-peak; the oscillation period for both fatiguing and threshold detection stimuli was $2.6 \mathrm{sec}$. Variability is indicated by one standard deviation either side of mean. 
than six observers, all of whom had participated in Experiment 1, were employed. Instead of only one postfatigue threshold determination, three postfatigue threshold determinations were performed at 2, 5, and $10 \mathrm{~min}$. Four rather than 10 tracking reversals were used to determine postfatigue thresholds. Each threshold determination required approximately $2 \mathrm{~min}$ to complete; consequently, the " 2 -min" postfatigue threshold was determined between 1 and 3 min following fatiguing stimulus termination, the " 5 -min" postfatigue threshold was determined between 4 and $6 \mathrm{~min}$, and the " 10 -min" threshold was determined between 9 and $11 \mathrm{~min}$.

As in Experiment 1, each fatiguing stimulus duration was replicated four times for each observer; therefore, 16 experimental sessions were required for each observer to complete the experiment.

\section{Results}

Sustained linear oscillation resulted in increased motion detection thresholds, confirming the results of Experiment 1. This motion detection threshold shift gradually subsided during the recovery period, and the thresholds returned to approximately prefatigue levels within a relatively brief period. Figure 2 , which illustrates thresholds following exposure to $5 \mathrm{~min}$ of sustained oscillation plotted on semi-log coordinates, indicates that this recovery of motion sensitivity approximates an exponential function of time. By extrapolation, complete recovery (return to the prefatigue threshold level) would have taken place about $13 \mathrm{~min}$ following termination of the 5-min sustained oscillation. Differences among absolute threshold levels as a function of postfatigue recovery time $(2,5$, and $10 \mathrm{~min})$ were statistically significant, $F(3,12)=65.41, p<.0001$. Differences among thresholds as a function of fatiguing stimulus duration $(0,2,5$, and $10 \mathrm{~min})$ were also statistically significant, $F(3,12)=59.71, \mathrm{p}<.0001$.

\section{Discussion}

The results of Experiments 1 and 2 demonstrated that thresholds for motion detection were increased

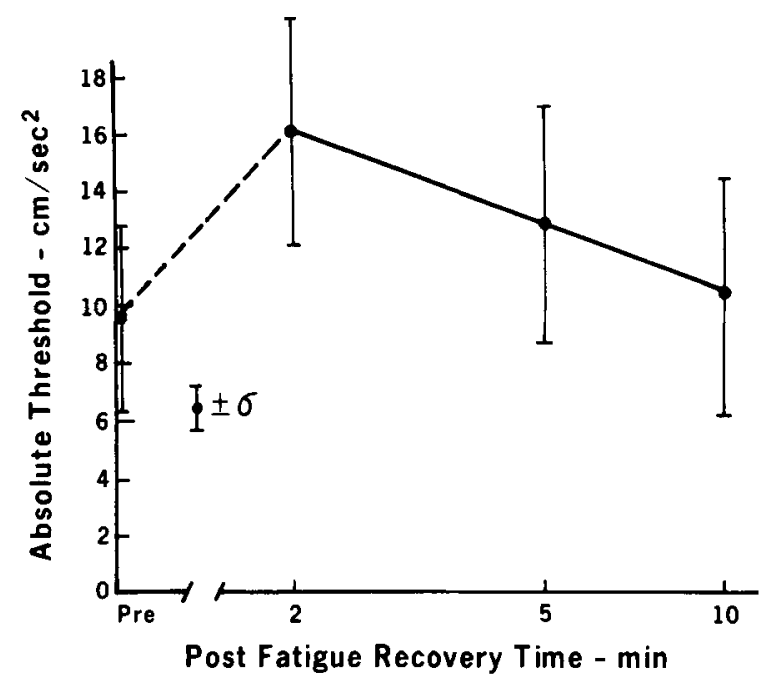

Figure 2. Recovery from fatigue produced by intense, oscillating linear acceleration. Abscissa indicates time following termination of 5-min fatiguing stimulus. Ordinate indicates peak-to-peak acceleration thresholds in $\mathrm{cm} / \mathrm{sec}^{2}$.

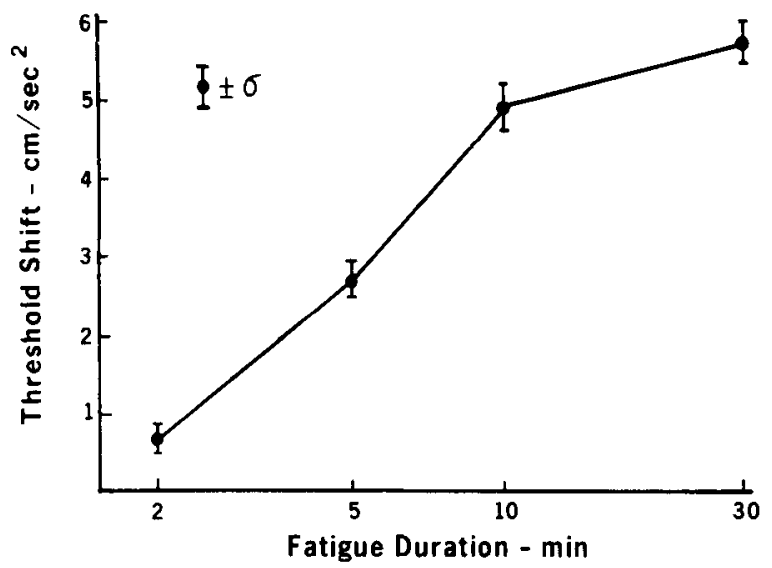

Figure 3. Shifts in linear motion detection thresholds following sustained oscillation for periods up to $30 \mathrm{~min}$.

following sustained oscillation and that these threshold increases were transient. The data illustrated in Figure 1 suggest that the function describing the relationship between fatigue duration and the temporary threshold shift is negatively accelerated; that is, increasing fatigue duration beyond $10 \mathrm{~min}$ should result in relatively small increases in threshold shift. Experiment 3 was designed to evaluate this suggestion by extending the duration of sustained oscillation to $30 \mathrm{~min}$.

\section{EXPERIMENT 3 LONG-DURATION FATIGUE}

\section{Method}

The methods employed in Experiment 3 were similar to those employed in Experiments 1 and 2. Four observers were used in this experiment; two of these observers had participated in the previous experiments and two were new. Two were males and two females. The age range was 20 to 26 years. Thresholds were determined prior to sustained oscillation and 2,5 , and $10 \mathrm{~min}$ following exposure to this fatiguing stimulus, as in Experiment 2. Four fatiguing stimulus durations were employed: $2,5,10$, and $30 \mathrm{~min}$. The fatiguing stimulus consisted of sustained oscillation at $.26 \mathrm{~Hz}$ and $468 \mathrm{~cm} / \mathrm{sec}^{2}$ peak-to-peak. As in the previous experiments, each observer was exposed to each fatiguing stimulus duration four times, and the order of durations across sessions was randomized. Consequently, 16 experimental sessions were required for each observer to complete the experiment.

The orientation of the observers in the parallel swing during Experiment 3 differed from that used in Experiments 1 and 2. For all of the threshold determinations, the observer's head was in the $0^{\circ}$ orientation (utricular main parts in the horizontal plane with the otoliths above the macular surface). However, during sustained oscillation the observers were placed in the $180^{\circ}$ orientation (utricular main parts in the horizontal plane with the otoliths suspended below the macular surface).

\section{Results}

Figure 3 illustrates the magnitude of threshold shift obtained 2 min after fatiguing stimulus termination as a function of duration. The curve indicates that increased threshold shifts were obtained with longer fatiguing stimulus durations. Differences among threshoids as a function of duration were statistically significant, $F(3,9)=32.46, p<.001$. 
These data also confirm the suggestion that the function relating threshold shift to fatiguing stimulus duration is negatively accelerated; increasing duration beyond 10 min produced only a small increase in the magnitude of the threshold shift. The recovery curves obtained in Experiment 3 were similar to those obtained in Experiment 2; the thresholds returned to nearly prefatigue levels within $10 \mathrm{~min}$ after fatigue termination.

\section{Discussion}

The threshold shifts illustrated in Figure 3 are somewhat smaller than those illustrated in Figure 1. This difference between the results of Experiments 1 and 3 is attributed to a general increase in the sensitivity and consistency of the observers and a small lowering of their thresholds with increased experience in the experiment.

Experiment 3 represented a preliminary attempt to demonstrate that the sustained oscillation affected the vestibular receptors rather than skin pressure receptors. The rationale underlying this demonstration is as follows. Suppose the threshold shifts following sustained oscillation resulted from stimulation of skin receptors. During the prefatigue and postfatigue threshold determinations, the observer was in the prone position $\left(0^{\circ}\right.$ orientation). If the skin receptors mediated the motion perception reported by the observer, the major skin receptors involved should have been those located on the ventral surface of the observer's body. During sustained oscillation, the observer was placed in the supine position $\left(180^{\circ}\right.$ orientation); the skin receptors most affected by the fatiguing stimulus should have been those located on the dorsal side of the body. Therefore, the fatiguing stimulus should have had little or no effect on ventral surface skin receptors and no threshold shift should have been observed. On the other hand, suppose that the main parts of the utricular maculae were primarily responsible for the motion perception reported by the observers and that the stimulus force component perpendicular to the macular surface (shearing force) allowed the observers to detect the linear oscillation. In both the $0^{\circ}$ orientation, during pre- and postfatigue threshold determinations, and the $180^{\circ}$ orientation, during exposure to the fatiguing stimulus, the shearing force acting on the maculae should have been essentially the same. Therefore, a threshold shift should have been induced by the sustained oscillation. The results of Experiment 3 support the view that the motion perception reported by the observers resulted from stimulation of the utricles and that the fatiguing stimulus affected these receptors.

The issue of the particular receptors involved in the motion perception and affected by the fatiguing stimulus was explored further in Experiment 4. In this experiment, as in Experiment 3, the observer was placed in the $0^{\circ}$ orientation during threshold determination; five orientations ranging from $0^{\circ}$ to $180^{\circ}$ were used during presentation of the fatiguing stimulus.

\section{EXPERIMENT 4 ORIENTATION}

\section{Method}

The procedure employed in Experiment 4 was similar to that used in the previous experiments. Four observers (two male, two female), all of whom were experienced in performing motion threshold judgments as a result of participation in at least one of the previous experiments, were used in Experiment 4. Thresholds were determined following the same procedure as used in Experiment 3; the fatiguing stimulus was 5 min of sustained oscillation at $.26 \mathrm{~Hz}$ and a peak-to-peak displacement of $176 \mathrm{~cm}$.

During exposure to sustained oscillation, the observers were placed in one of five orientation: $0^{\circ}, 45^{\circ}, 90^{\circ}, 135^{\circ}$, and $180^{\circ}$. An experimental session consisted of the pre- and postfatigue threshold determinations and sustained oscillation in one of the five orientations. Each observer was exposed to the fatiguing stimulus in each of the orientations four times; the order of presentation of the orientations was randomized. At least $24 \mathrm{~h}$ elapsed between experimental sessions.

\section{Results}

The results of Experiment 4 are summarized in Figure 4. As in the previous experiments, $5 \mathrm{~min}$ of sustained oscillation produced a notable threshold shift when the thresholds were determined in the $0^{\circ}$ orientation and the fatiguing stimulus was presented in either the $0^{\circ}$ or the $180^{\circ}$ orientation. Furthermore,

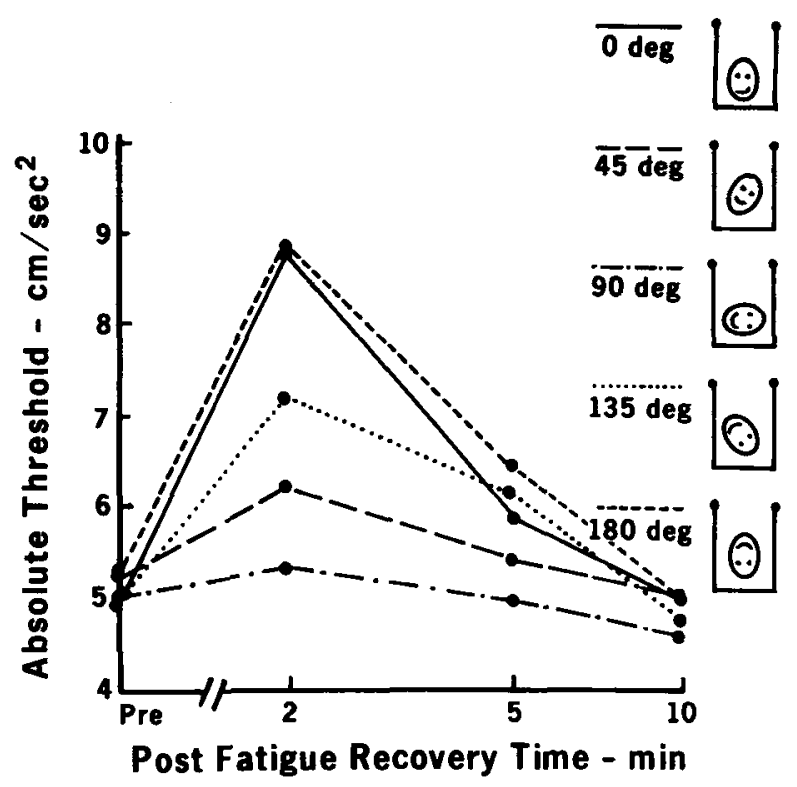

Figure 4. Increases in absolute motion detection thresholds and recovery following exposure to 5 -min fatiguing stimulus in various orientations. Thresholds were determined in the $0^{\circ}$ orientation; fatiguing stimulus exposure was in the $0^{\circ}, 45^{\circ}, 90^{\circ}, 135^{\circ}$, and $180^{\circ}$ orientations. 
as in the previous experiments, recovery of the threshold to the prefatigue level was nearly complete after $10 \mathrm{~min}$. The most interesting aspect of the data, however, is concerned with the magnitude of change in threshold following sustained oscillation in the different orientations: large threshold shifts following sustained oscillation were obtained when the observers were placed in the $0^{\circ}$ and $180^{\circ}$ orientations; little threshold shift was noted following exposure to sustained oscillation in the $45^{\circ}, 90^{\circ}$, or $135^{\circ}$ orientations. Differences among the threshold shifts at 2 min postfatigue as a function of orientation were statistically significant, $\mathrm{F}(4,12)=8.96, \mathrm{p}<.005$.

\section{Discussion}

The results of Experiment 4 support the view that vestibular receptors rather than skin receptors are responsible for the observers' responses, following the rationale presented in the discussion of Experiment 3 . The observations that large threshold shifts were obtained following sustained oscillation exposure in the $0^{\circ}$ and $180^{\circ}$ orientations, whereas little or no threshold shift was obtained following exposure in the $45^{\circ}, 90^{\circ}$, or $135^{\circ}$ orientations, support the view that the main parts of the utricular maculae are responsible for the motion perception reported by the observers.

\section{EXPERIMENT 5 CENTRIFUGATION}

Macadar, Wolfe, O'Leary, \& Segundo (1975) examined responses from otolith neurons in the isolated labyrinth of the ray and suggested that these neurons could be divided into phasic, phasic-tonic, and tonic units on the basis of their response characteristics. Several other investigators have noted similar distinctions in the mammalian vestibular nerve and vestibular nuclei (Adrian, 1943; Melvill-Jones, 1968; Schor, 1974). Fernandez and Goldberg (1976) found that most of the squirrel monkey otolith neurons they examined could be classified as phasictonic, indicating that these units exhibited some adaptation to sustained linear acceleration but did continue to respond throughout the duration of the linear acceleration.

Suppose that the neurons mediating the motion detection thresholds obtained in the previous experiments were of the phasic-tonic type. Suppose further that the interpretation offered to explain the results of the previous experiments were correct, i.e., that the sustained oscillation resulted in fatigue of the vestibular receptor. Finally, suppose that sustained unidirectional linear acceleration could fatigue these sensory receptors in a manner analogous to sustained, oscillating linear oscillation. These suppositions would lead to the hypothesis that sustained unidirectional linear acceleration resulting from centri- fugation should produce a temporary threshold shift for motion detection on the parallel swing. This hypothesis was examined in Experiment 5.

\section{Method}

Five observers (three female, two male) participated in Experiment 5. Of these five, one was new and the other four had participated in at least one of the previous experiments.

Motion thresholds were obtained with the parallel swing, as described previously. Sustained, unidirectional linear acceleration (fatiguing stimulus) was obtained with a centrifuge. The centrifuge consisted of a $122-\mathrm{cm}$ radius circular platform which was driven through a reduction gear by a 10 -hp, variable-speed $\mathrm{dc}$ motor. A servo regulator maintained the centrifuge rotation rate at a preset level. A restraint device was mounted on the edge of the centrifuge and the observer's head was positioned $98 \mathrm{~cm}$ from the axis of rotation with the main parts of the utricular maculae oriented parallel to the vector which was the resultant of the linear acceleration generated by the centrifuge and the linear acceleration due to gravity. The centrifugal acceleration of $1.73 \mathrm{G}$, which was produced by a rotation rate of $40 \mathrm{rpm}$, summed with the acceleration due to gravity to produce an acceleration of $2.0 \mathrm{G}$ at the center of the observer's head. Rotation rate was increased from 0 to $40 \mathrm{rpm}$ over a period of $1 \mathrm{~min}$; deceleration from 40 to $0 \mathrm{rpm}$ required $30 \mathrm{sec}$.

Motion detection thresholds were obtained as described for Experiment 4, with the exception that postfatigue (postcentrifugation) thresholds were performed at 4,7 , and $10 \mathrm{~min}$. After the prefatigue threshold had been determined, the observer was placed in the centrifuge and exposed to 5 min of $2-\mathrm{G}$ acceleration. At the termination of centrifugation, the observer was transported on a cart back to the parallel swing, which was located in another room. Working rapidly, it was consistently possible to obtain the initial postfatigue threshold determination $4 \mathrm{~min}$ after termination of centrifugation. The observers were placed in the parallel swing in either the $0^{\circ}$ or the $180^{\circ}$ orientation. The reactions of each observer to centrifugation were examined four times for each of the two parallel swing orientations, yielding a total of eight trials from each observer. If the prefatigue thresholds were determined in the $0^{\circ}$ orientation, postfatigue thresholds were also obtained in the $0^{\circ}$ orientation; if the observer's prefatigue thresholds were obtained in the $180^{\circ}$ orientation, postfatigue thresholds were obtained in that same orientation.

\section{Results}

No evidence of fatigue was obtained in Experiment 5 . Across the 40 experimental sessions, motion detection thresholds were greater $4 \mathrm{~min}$ following centrifugation than before centrifugation in 22 cases. Given the null hypothesis (probability of increase in threshold following rotation $=.5$ ), the probability of observing motion detection threshold increases in at least 22 out of 40 cases is .412 . Consequently, the null hypothesis that these observations occurred by chance alone cannot be rejected. When only the data for the $0^{\circ}$ orientation were examined, it was noted that in 13 of 20 possible cases the threshold was greater $4 \mathrm{~min}$ postfatigue than prior to fatigue. The likelihood of obtaining this result by chance $(p=.132)$ is greater than the normally accepted level for statistical significance; again, the null hypothesis cannot be rejected.

\section{Discussion}

The results of Experiment 5 offer several possibil- 
ities for interpretation. Possibly, motion detection in the parallel swing is mediated by a different set of receptors than those effected by sustained unidirectional linear acceleration. Another possibility is that the centrifugation affected the receptor's responses only for motion in a particular direction; since the motion in the parallel swing can be considered at least bidirectional, motion detection thresholds in this apparatus were unaffected. This second possibility is consistent with the report of one of the observers who indicated that he was unable to detect motion easily in one direction, but that motion perception in the opposite direction was unaffected.

A supplementary study was carried out to examine the effects of $10 \mathrm{~min}$ of centrifugation. Only two observers were used, and each observer was examined four times in the $180^{\circ}$ orientation. The results indicated increased motion detection thresholds following centrifugation in four of eight cases. Again, these observations appear to be accounted for by chance alone.

\section{GENERAL DISCUSSION}

\section{Thresholds}

The thresholds for self-motion reports employing various procedures and under a variety of stimulus conditions have been reviewed by Guedry (1974). The observers in the present study were asked to indicate when they perceived that they were moving. For the five experiments, the mean prefatigue motion detection thresholds across observers ranged from 3 to $9 \mathrm{~cm} / \mathrm{sec}^{2}$. The thresholds were highest in the initial experiment and gradually declined across the series as the observers gained more experience in the parallel swing. Throughout the investigation, catch trials were used to insure that the observers were responding to "real" motion. These thresholds are lower than those reported by previous investigators, including Walsh $(1962,1964)$. It is not clear that Walsh's observers employed the same response criteria as was employed by the observers in the present investigation. Other experiments undertaken at Miami University indicate that the stimulus amplitudes required for an observer to accurately detect motion reversal in a parallel swing are two to three times greater than those levels needed to elicit basic reports of motion.

\section{Fatigue or Adaptation?}

The results of Experiments 1-4 are interpreted as indicating that sustained linear oscillation can fatigue the receptors of the vestibular system. In this section, the fatigue interpretation is supported by relating the experiments described herein to previous fatigue experiments in the field of audition.

Following Hood's (1950) initial proposal, several response characteristics have been cited by auditory investigators to distinguish adaptation from fatigue. Among the distinctions are the following: experimental procedures, characteristics of the changeinducing stimulus, the temporal characteristics of the threshold changes, qualitative changes in the loudness function, and several physiological alterations. With regard to experimental procedure, adaptation studies ordinarily determine alteration in responsiveness during stimulus presentation, whereas fatigue studies examine changes after the termination of some moderate or intense stimulus. Fatigue studies employ moderate-to-intense levels of stim ilation to induce response change, while adaptation studies employ weak-to-moderate levels of stimulation. The temporal course of adaptation is relatively brief: adaptation is usually completely developed within $3 \mathrm{~min}$ and recovery occurs within 1-2 min. On the other hand, fatigue can continue to increase for hours, and the time required for recovery from fatigue is dependent upon the magnitude of the initial change. Adaptation apparently results in a less-thannormal growth of the loudness function, whereas fatigue results in a greater-than-normal loudness function growth (recruitment). Finally, physiological changes that distinguish adaptation from fatigue include characteristics of the cochlear potential and chemical characteristics of the endolymph. These distinctions were discussed by Elliot and Fraser (1970).

The experimental procedure and the temporal characteristics of the changes obtained in the experiments described in this paper are analogous to those used in auditory fatigue studies. The procedures employed in Experiments 1-5 followed the standard method of auditory temporary threshold shift experiments. As illustrated by Figures 1, 3, and 4, the motion detection thresholds continue to increase as a function of fatigue duration for periods of up to $30 \mathrm{~min}$. Also as illustrated in Figure 4, the time required for recovery to prefatigue levels is dependent upon the magnitude of the initial threshold shift. These observations support the view that the results of Experiments 1-4 should be interpreted as indicating vestibular fatigue.

It is difficult to determine whether the stimulus used in the sustained oscillation experiments should be considered as weak, moderate, or intense. Certainly, the exact frequency-intensity stimulus characteristics employed in the sustained oscillation experiments would not have been encountered ordinarily by the ancestors of modern human beings. Parker, Covell, \& von Gierke (1968) obtained evidence of otoconia displacement in guinea pigs following vibration at $8 \mathrm{~Hz}$ and 1-2 $\mathrm{G}$ for periods of 4 to $6 \mathrm{~h}$; no evidence of behavioral loss was obtained in that investigation. However, the differences in the frequency characteristics of the stimuli plus the apparent discrepancies between Parker's observations with guinea pigs and Igarashi and Nagaba's (1968) observations with 
monkeys make difficult application of the vestibular damage observations to the present investigation.

Behavioral studies on the magnitude of perceived displacement as a function of variation of stimulus intensity and neurophysiological studies of changes in the vestibular pathway following fatigue are suggested by the results of the present investigation. Preliminary studies of the magnitude of perceived displacement are being pursued at Miami University; however, insufficient data have been obtained to report here.

In sum, the results of the experiments described in this paper are analogous to the results of auditory research that has been interpreted as indicating fatigue of the auditory receptor or afferent pathway. Because previous vestibular investigations, particularly those dealing with adaptation or habituation, have not employed procedures similar to those used in Experiments 1-5, the fatigue interpretation proposed here can be neither refuted nor supported by this previous vestibular research.

\section{REFERENCES}

ADrian, E. D. Discharges from vestibular receptors in the cat. Journal of Physiology (London), 1943, 101, 389.407.

Collins, W. E. Arousal and vestibular habituation. In $\mathbf{H}$. $\mathrm{H}$. Kornhuber (Ed.), Handbook of sensory physiology (VI/2). Berlin: Springer-Verlag, 1974. (a)

Colliss, W. E. Habituation of vestibular responses with and without visual stimulation. In $H$. H. Kornhuber (Ed.), Handbook of sensory physiology (VI/2). Berlin: SpringerVerlag, 1974. (b)

Elliotr, D. N., \& Fraser, W. R. Fatigue and adaptation. In J. V. Tobias (Ed.), Foundations of modern auditory theory (Vol. 1). New York: Academic Press, 1970.

Fernandez, C., \& Goldberg, J. M. Physiology of peripheral neurons innervating otolith organs of the squirrel monkey. I. Response to static tilts and to long-duration centrifugal force. Journal of Neurophysiology, 1976, 39, 970-984.
Guedry, F. E. Psychophysics of vestibular sensation. In H. H. Kornhuber (Ed.), Handbook of sensory physiology (VI/2). Berlin: Springer-Verlag, 1974.

Hood, J. D. Studies of auditory fatigue and adaptation. Acta Otolaryngologica (Stockholm), 1950, Suppl. 92.

IgARASHI, M. \& NAGABA, M. Vestibular end-organ damage in squirrel monkeys after exposure to intensive linear acceleration. In Third symposium on the role of the vestibular organs in space exploration, NASA SP-152. Washington, D.C: National Aeronautics and Space Administration, 1968.

Macadar, O., Wolfe, G. E., O'Leary, D. P., \& Segundo, J. P. Response of the elasmobranch utricle to maintained spatial orientation, transitions and jitter. Experimental Brain Research, $1975,22,1-12$.

MeLvill-Jones, G. Neural reflection of vestibular organs in space exploration, NASA SP-152. Washington, D.C: National Aeronautics and Space Administration, 1968.

PARKeR, D. E. Interactions between electrical and mechanical vestibular stimulation: Observations in rabbits and men. Journal of Experimental Psychology, 1970, 84, 96-104.

Parker, D. E., Covell, W. P., \& von Gierke, H. E. Exploration of vestibular damage in guinea pigs following mechanical stimulation. Acta Otolaryngologica (Stockholm), 1968, Suppl. 239.

Reason, J. T., \& Graybiel, A. Progressive adaptation to Coriolis accelerations associated with $1 \mathrm{rpm}$ increments in the velocity of the Slow Rotation Room. Aerospace Medicine, $1970,41,73-79$.

Schöne, H., \& Lechner-Steinleitner, S. The effect of preceding tilt on the perceived vertical (hysteresis in perception of the vertical). Acta Otolaryngologica (Stockholm), 1978, 85, 68-73.

Schor, R. H. Response of cat vestibular neurons to sinusoidal role tilt. Experimental Brain Research, 1974, 20, 347-362.

WALSH, E. G. The perception of rhythmically repeated linear motion in the horizontal plane. British Journal of Psychology, $1962,53,439-445$

WALsH, E. G. The perception of rhythmically repeated linear motion in the vertical plane. Quarterly Journal of Experimental Physiology, 1964, 49, 58-65.

(Received for publication December 19, 1977; revision accepted April 10, 1978.) 